\title{
Growth rate for endomorphisms of finitely generated nilpotent groups
}

\author{
Alexander Fel'shtyn, Jang Hyun Jo and Jong Bum Lee* \\ Communicated by Evgenii I. Khukhro
}

\begin{abstract}
We prove that the growth rate of an endomorphism of a finitely generated nilpotent group is equal to the growth rate of the induced endomorphism on its abelianization, generalizing the corresponding result for an automorphism in [T. Koberda, Entropy of automorphisms, homology and the intrinsic polynomial structure of nilpotent groups, in: In the Tradition of Ahlfors-Bers. VI, Contemp. Math. 590, American Mathematical Society, Providence (2013), 87-99].
\end{abstract}

\section{Introduction}

In the present paper, we study purely algebraic notions of growth rate and entropy for an endomorphism of a finitely generated group.

Let $\pi$ be a finitely generated group with a system $S=\left\{s_{1}, \ldots, s_{n}\right\}$ of generators. Let $\phi: \pi \rightarrow \pi$ be an endomorphism. For any $\gamma \in \pi$, let $L(\gamma, S)$ be the length of the shortest word in the letters $S \cup S^{-1}$ which represents $\gamma$. Then the growth rate of $\phi$ is defined [2] to be

$$
\operatorname{GR}(\phi):=\sup \left\{\limsup _{k \rightarrow \infty} L\left(\phi^{k}(\gamma), S\right)^{1 / k} \mid \gamma \in \pi\right\}
$$

For each $k>0$, we put

$$
L_{k}(\phi, S):=\max \left\{L\left(\phi^{k}\left(s_{i}\right), S\right) \mid i=1, \ldots, n\right\} .
$$

It is known for example from [2, Proposition 1] that

$$
\mathrm{GR}(\phi)=\lim _{k \rightarrow \infty} L_{k}(\phi, S)^{1 / k}=\inf _{k}\left\{L_{k}(\phi, S)^{1 / k}\right\},
$$

The work of the first-named author is funded by the Narodowe Centrum Nauki of Poland (NCN) (grant No. 2016/23/G/ST1/04280 (Beethoven 2)). The second-named author was supported by Basic Science Research Program through the National Research Foundation of Korea (NRF) funded by the Ministry of Education (NRF-2016R1D1A1B03932318). The third-named author was supported by Basic Science Research Program through the National Research Foundation of Korea (NRF) funded by the Ministry of Education (NRF-2016R1D1A1B01006971). 
and the algebraic entropy of $\phi$ is by definition $h_{\mathrm{alg}}(\phi):=\log \operatorname{GR}(\phi)$. The growth rate and hence the algebraic entropy of $\phi$ are well-defined, i.e., independent of the choice of a set of generators [7, p. 114]. It is immediate from the definition that the growth rate and the algebraic entropy for an endomorphism of a group are invariants of conjugacy of group endomorphisms. Furthermore, for any inner automorphism $\tau_{\gamma_{0}}$ by $\gamma_{0}$, we have $\operatorname{GR}\left(\tau_{\gamma_{0}} \phi\right)=\operatorname{GR}(\phi)$ and $h_{\mathrm{alg}}\left(\tau_{\gamma_{0}} \phi\right)=h_{\mathrm{alg}}(\phi)$ ([7, Proposition 3.1.10]).

Consider a continuous map $f$ on a compact connected manifold $M$, and consider a homomorphism $\phi$ induced by $f$ of the group of covering transformations on the universal cover of $M$. Then the topological entropy $h_{\text {top }}(f)$ is defined. We refer to [7] for background. Several authors, among them R. Bowen in [2] and A. Katok in [8], have proved that the topological entropy $h_{\text {top }}(f)$ of $f$ is at least as large as the algebraic entropy $h_{\mathrm{alg}}(\phi)=h_{\mathrm{alg}}(f)$ of $\phi$ or $f$.

The problem of determining the growth rate of a group endomorphism, initiated by R. Bowen in [2], is now an area of active research (see detailed description in [5, $10]$ and references therein). For known properties of the growth of automorphisms of free groups, we refer to $[1,12,13]$.

The purpose of this paper is first to study the growth rate of an endomorphism on a finitely generated nilpotent group. In [10, Theorem 1.2], it was proven that the growth rate of an automorphism of a finitely generated nilpotent group is equal to the growth rate of the induced automorphism on its abelianization. Our main result is a generalization of this result of [10] from automorphisms to endomorphisms, using completely different arguments. In Section 2, we recall some known results about the growth rate of a group endomorphism, sometimes correcting them. In Section 3, we refine the calculation in [2] of the growth rate for an endomorphism of a finitely generated torsion-free nilpotent group and prove that the growth rate is an algebraic integer.

Let $\pi$ be a finitely generated torsion-free nilpotent group, and let $G$ be its Malcev completion. Let $\phi$ be an endomorphism of $\pi$. Then $\phi$ extends uniquely to a Lie group homomorphism $D$ of $G$, called the Malcev completion of $\phi$. We call its differential $D_{*}$ the linearization of $\phi$. The main results are the following.

Theorem 3.3. Let $\phi: \pi \rightarrow \pi$ be an endomorphism on a finitely generated torsionfree nilpotent group $\pi$. Let $G$ be the Malcev completion of $\pi$. Then the linearization $D_{*}:(S \rightarrow$ S of $\phi$ can be expressed as a lower triangular block matrix with diagonal blocks $\left\{D_{j}\right\}$ so that

$$
\operatorname{GR}(\phi)=\max _{j \geq 1}\left\{\operatorname{sp}\left(D_{j}\right)^{1 / j}\right\} .
$$

In particular, $\mathrm{GR}(\phi)$ is an algebraic integer. 
Theorem 3.7. Let $\phi: \pi \rightarrow \pi$ be an endomorphism on a finitely generated torsionfree nilpotent group $\pi$ with Malcev completion D. Then

$$
\operatorname{GR}(\phi)=\operatorname{GR}\left(\phi_{\mathrm{ab}}\right),
$$

where $\phi_{\mathrm{ab}}: \pi /[\pi, \pi] \rightarrow \pi /[\pi, \pi]$ be the endomorphism induced by $\phi$. Hence we have $\operatorname{GR}(\phi)=\operatorname{sp}\left(D_{1}\right) \leq \operatorname{sp}\left(D_{*}\right)$.

\section{Preliminaries}

We shall assume in this article that all groups are finitely generated unless otherwise specified. For a given endomorphism $\phi: \pi \rightarrow \pi$, if $\pi^{\prime}$ is a $\phi$-invariant subgroup of $\pi$, we denote by $\phi^{\prime}=\left.\phi\right|_{\pi^{\prime}}$ the restriction of $\phi$ to $\pi^{\prime}$. If, in addition, $\pi^{\prime}$ is a normal subgroup, we denote by $\hat{\phi}$ the endomorphism on $\pi / \pi^{\prime}$ induced by $\phi$. Then the following are known; see for example [2,5].

- $\operatorname{GR}\left(\phi^{k}\right)=\operatorname{GR}(\phi)^{k}$ for $k>0$.

- $\operatorname{GR}(\hat{\phi}) \leq \operatorname{GR}(\phi)$.

- $\operatorname{GR}(\phi) \leq \max \left\{\operatorname{GR}\left(\phi^{\prime}\right), \operatorname{GR}(\hat{\phi})\right\}$.

- Let $\phi: \mathbb{Z}^{n} \rightarrow \mathbb{Z}^{n}$ be an endomorphism yielding an integer matrix $D$. Then we have $\operatorname{GR}(\phi)=\operatorname{sp}(D)$, the maximum of the absolute values of the eigenvalues of $D$.

Let $S^{\prime}$ be a finite set of generators for $\pi^{\prime}$, and let $\hat{S}$ be a finite set of generators for the quotient group $\pi / \pi^{\prime}$. Then it is possible to extend $S^{\prime}$ to a finite set $S$ of generators for $\pi$ so that $S$ is projected onto $\hat{S}$ under the projection $\pi \rightarrow \pi / \pi^{\prime}$. For any $\gamma \in \pi^{\prime}$, it is true that $L\left(\gamma, S^{\prime}\right) \geq L(\gamma, S)$.

Consider the concentric balls $B(n)=\{\gamma \in \pi \mid L(\gamma, S) \leq n\}$ for all $n>0$, and the distortion function of $\pi^{\prime}$ in $\pi$ which is defined as

$$
\Delta_{\pi^{\prime}}^{\pi}(n):=\max \left\{L\left(\gamma, S^{\prime}\right) \mid \gamma \in \pi^{\prime} \cap B(n)\right\} .
$$

The notion of distortion of a subgroup was first introduced by M. Gromov in [6]. We refer to [3] for our discussion. For two functions $f, g: \mathbb{N} \rightarrow \mathbb{N}$, we say that $f \preccurlyeq g$ if there exists $c>0$ such that such that $f(n) \leq \operatorname{cg}(c n)$ for all $n>0$. We say that two functions are equivalent, written $f \approx g$, if $f \preccurlyeq g$ and $g \preccurlyeq f$. The subgroup $\pi^{\prime}$ of $\pi$ is undistorted if $\Delta_{\pi^{\prime}}^{\pi}(n) \approx n$. The following facts about distortion can be found in [3].

- If $\pi^{\prime}$ is infinite, then it is true that $n \preccurlyeq \Delta_{\pi^{\prime}}^{\pi}(n)$.

- If $\left[\pi: \pi^{\prime}\right]<\infty$, then $\pi^{\prime}$ is undistorted in $\pi$. 
Assume $\Delta_{\pi^{\prime}}^{\pi}(n) \preccurlyeq n$. By definition, there exists $c>0$ such that $\Delta_{\pi^{\prime}}^{\pi}(n) \leq c^{2} n$ for all $n>0$. For any $\gamma \in \pi^{\prime}$, let $n=L(\gamma, S)$. Then

$$
L\left(\gamma, S^{\prime}\right) \leq \Delta_{\pi^{\prime}}^{\pi}(n) \leq c^{2} n=c^{2} L(\gamma, S) .
$$

Thus $L(\gamma, S) \leq c^{2} L(\gamma, S)$ for all $\gamma \in \pi^{\prime}$. This inequality implies that, for all $k>0$,

$$
\begin{aligned}
L_{k}\left(\phi^{\prime}, S^{\prime}\right) & =\max \left\{L\left(\phi^{\prime k}\left(\gamma_{i}\right), S^{\prime}\right) \mid \gamma_{i} \in S^{\prime}\right\} \\
& \leq c^{2} \max \left\{L\left(\phi^{\prime k}\left(\gamma_{i}\right), S\right) \mid \gamma_{i} \in S^{\prime}\right\} \leq c^{2} L_{k}(\phi, S),
\end{aligned}
$$

and so $\operatorname{GR}\left(\phi^{\prime}\right) \leq \operatorname{GR}(\phi)$. Consequently, we have the following lemma.

Lemma 2.1 ([5, Corollary 3.1]). Let $\phi$ be an endomorphism of $\pi$. If $\pi^{\prime}$ is a $\phi$-invariant undistorted subgroup in $\pi$, then $\operatorname{GR}\left(\phi^{\prime}\right) \leq \mathrm{GR}(\phi)$; hence if, in addition, $\pi^{\prime}$ is a normal subgroup of $\pi$, then $\operatorname{GR}(\phi)=\max \left\{\operatorname{GR}\left(\phi^{\prime}\right), \operatorname{GR}(\hat{\phi})\right\}$.

Proof. Since $\pi^{\prime}$ is undistorted in $\pi$, we have from the definition that $\Delta_{\pi^{\prime}}^{\pi}(n) \preccurlyeq n$. Now the proof follows from the above observation.

Remark 2.2. Note also the following.

- If $\pi^{\prime}$ is of finite index in $\pi$, then $\pi^{\prime}$ is undistorted, and hence $\operatorname{GR}\left(\phi^{\prime}\right) \leq \operatorname{GR}(\phi)$. Example 2.4 shows that the inequality can be strict. Thus [2, Proposition 1(3)] (see also [5, Theorem 3.1]) is not correct.

- If $\operatorname{GR}(\phi)<\operatorname{GR}\left(\phi^{\prime}\right)$, then $\pi^{\prime}$ is distorted, and $\pi^{\prime}$ is not of finite index in $\pi$.

Lemma 2.3. Let $\phi$ be an endomorphism of $\pi$. If $\operatorname{GR}(\phi)<1$, then $\operatorname{GR}(\phi)=0$ and $\phi$ is an eventually trivial endomorphism, and vice versa.

Proof. Let $\rho=\operatorname{GR}(\phi)$, and let $\epsilon=1-\rho>0$. Since $\lim _{m \rightarrow \infty} L_{m}(\phi, S)^{1 / m}=\rho$, there exists $N>0$ such that, for all $m \geq N$, we have $L_{m}(\phi, S)^{1 / m}-\rho<\epsilon$;

$$
L_{m}(\phi, S)^{1 / m}<1 \Longrightarrow L_{m}(\phi, S)<1 \Longrightarrow L_{m}(\phi, S)=0
$$

because $L_{m}(\phi, S)$ is a nonnegative integer. This implies that $\rho=0$ and the endomorphism $\phi^{N}$ is trivial or $\phi$ is eventually trivial. The converse is obvious.

Example 2.4. Let $\pi=\mathbb{Z} \times \mathbb{Z}_{2}$ with generators $\alpha$ and $\beta$ such that $\beta^{2}=1$. Consider an endomorphism $\phi$ of $\pi$ defined by $\phi(\alpha)=1$ and $\phi(\beta)=\beta$. Observing that

$$
\begin{aligned}
L_{n}(\phi, S) & =\max \left\{L\left(\phi^{n}(\alpha), S\right), L\left(\phi^{n}(\beta), S\right)\right\} \\
& =\max \{L(1, S), L(\beta, S)\}=\max \{0,1\}=1,
\end{aligned}
$$


we have $\operatorname{GR}(\phi)=1$. Similarly, we have $\operatorname{GR}\left(\left.\phi\right|_{\mathbb{Z}}\right)=0$ and $\operatorname{GR}\left(\left.\phi\right|_{\mathbb{Z}_{2}}\right)=1$. Notice further that $\mathbb{Z}_{2}$ is a distorted subgroup of $\pi$ because $\Delta_{\mathbb{Z}_{2}}^{\pi}(n)=1$ for all $n$.

Lemma 2.5. Let $\phi$ be an endomorphism of $\pi$.

(1) If $\pi^{\prime}$ is a $\phi$-invariant finite subgroup of $\pi$, then $\operatorname{GR}\left(\phi^{\prime}\right) \leq \operatorname{GR}(\phi)$.

(2) If, in addition, $\pi^{\prime}$ is a normal subgroup of $\pi$, then

$$
\operatorname{GR}(\phi)=\max \left\{\operatorname{GR}\left(\phi^{\prime}\right), \operatorname{GR}(\hat{\phi})\right\},
$$

and $\operatorname{GR}(\phi)=\operatorname{GR}(\hat{\phi})$ if and only if $\phi^{\prime}$ is eventually trivial or $\hat{\phi}$ is not eventually trivial.

Proof. If the $\phi$-invariant subgroup $\pi^{\prime}$ of $\pi$ is finite, then we can show easily that $\operatorname{GR}\left(\phi^{\prime}\right)$ is either 0 or 1 by taking a system of generators $S^{\prime}=\pi^{\prime}$ for $\pi^{\prime}$. We will show that $\operatorname{GR}\left(\phi^{\prime}\right) \leq \operatorname{GR}(\phi)$. We may assume that $\operatorname{GR}\left(\phi^{\prime}\right)=1$. This implies that there is an element $x \in \pi^{\prime}$ such that $\phi^{\prime n}(x) \neq 1$ for all $n>0$. Considering any system of generators for $\pi$ which contains $x$, we can see right away that $\operatorname{GR}(\phi) \geq 1=\operatorname{GR}\left(\phi^{\prime}\right)$.

Assume that $\pi^{\prime}$ is normal in $\pi$. If $\operatorname{GR}\left(\phi^{\prime}\right)=0$, then clearly $\operatorname{GR}(\phi)=\operatorname{GR}(\hat{\phi})$. On the other hand, if $\operatorname{GR}\left(\phi^{\prime}\right)=1$, then $\operatorname{GR}(\phi)=\operatorname{GR}(\hat{\phi})$ if and only if $\operatorname{GR}(\hat{\phi}) \geq 1$ if and only if $\hat{\phi}$ is not eventually trivial by Lemma 2.3 .

Remark 2.6. However, the above lemma is not true when $\pi^{\prime}$ is infinite; see Example 2.7. Note further that if $\operatorname{GR}(\phi)<\operatorname{GR}\left(\phi^{\prime}\right)$, then $\pi^{\prime}$ is infinite.

The following is a well-known example about subgroup distortion.

Example 2.7. Let $\pi$ be the Baumslag-Solitar group

$$
B(1, n):=\left\langle a, b \mid a^{-1} b a=b^{n}\right\rangle, \quad n>1 .
$$

Then $S=\{a, b\}$ is a generating set for $\pi$. Let $\pi^{\prime}=\langle b\rangle$, and let $S^{\prime}=\{b\}$. We observe that the subgroup $\pi^{\prime}$ of $\pi$ is distorted. In fact, since $b^{n^{k}}=a^{-k} b a^{k}$ for all $k>0$, we have that $L\left(b^{n^{k}}, S^{\prime}\right)=n^{k}$ and $L\left(b^{n^{k}}, S\right)=2 k+1$. If $\phi$ is an endomorphism of $\pi$ given by $\phi(b)=b^{n}$ and $\phi(a)=a$, then we can see that $\operatorname{GR}\left(\phi^{\prime}\right)=n$ and $\operatorname{GR}(\phi)=1$.

Example 2.4 shows that [2, Proposition 1(3)] is not correct in general, but it is almost true in the sense of Theorem 2.8. By modifying the argument of the proof of [5, Theorem 3.1], we have the following theorem. 
Theorem 2.8. Let $\phi$ be an endomorphism of $\pi$, and let $\pi^{\prime}$ be a $\phi$-invariant, finiteindex subgroup of $\pi$.

(1) If $\phi^{\prime}$ is not an eventually trivial endomorphism, then $\operatorname{GR}(\phi)=\mathrm{GR}\left(\phi^{\prime}\right)$.

(2) If $\phi^{\prime}$ is an eventually trivial endomorphism of $\pi^{\prime}$, then

$$
\operatorname{GR}\left(\phi^{\prime}\right)=0 \text { and } \operatorname{GR}(\phi)=0 \text { or } 1 .
$$

Moreover, $\operatorname{GR}(\phi)=0$ if and only if $\phi$ is an eventually trivial endomorphism of $\pi$.

Consequently, the equality $\operatorname{GR}(\phi)=\mathrm{GR}\left(\phi^{\prime}\right)$ holds except only for the case when $\phi^{\prime}$ is eventually trivial and $\phi$ is not eventually trivial. If this is the case, then $\operatorname{GR}\left(\phi^{\prime}\right)=0$ and $\operatorname{GR}(\phi)=1$.

Proof. Let $S^{\prime}=\left\{\gamma_{1}, \ldots, \gamma_{t}\right\}$ be a set of generators of $\pi^{\prime}$. Let $u=\left[\pi: \pi^{\prime}\right]$. Then we have $\pi=\delta_{1} \pi^{\prime} \cup \cdots \cup \delta_{u} \pi^{\prime}$ so that $S=\left\{\gamma_{1}, \ldots, \gamma_{t}, \delta_{1}, \ldots, \delta_{u}\right\}$ generates $\pi$. For any $j=1, \ldots, u$, there exists a unique $k_{j}$ such that $\phi\left(\delta_{j}\right) \in \delta_{k_{j}} \pi^{\prime}$. We denote

$$
p=\max _{1 \leq j \leq u}\left\{L\left(w_{j}, S^{\prime}\right) \mid \phi\left(\delta_{j}\right)=\delta_{k_{j}} w_{j} \in \delta_{k_{j}} \pi^{\prime}\right\} .
$$

Assume $p=0$. Then $\phi\left(\delta_{j}\right)=\delta_{k_{j}}$ for all $j=1, \ldots, u$. For each $j=1, \ldots, u$, we write $\phi^{m}\left(\delta_{j}\right)=\delta_{j_{m}}$. Hence it follows that $L\left(\phi^{m}\left(\delta_{j}\right), S\right)=0$ or 1 according to whether $\delta_{j_{m}}=1$ or $\delta_{j_{m}} \neq 1$.

Suppose that there is $N>0$ such that $\phi^{N}\left(\delta_{j}\right)=1$ for all $j=1, \ldots, u$ and hence $L\left(\phi^{m}\left(\delta_{j}\right), S\right)=0$ for all $m \geq N$. Since $\pi^{\prime}$ is undistorted in $\pi$, there exists some $c>0$ such that

$$
L\left(\gamma, S^{\prime}\right) \leq c^{2} \cdot L(\gamma, S) \quad \text { for all } \gamma \in \pi^{\prime} .
$$

It is clear that

$$
L(\gamma, S) \leq L\left(\gamma, S^{\prime}\right) \text { for all } \gamma \in \pi^{\prime}
$$

Thus

$$
\begin{aligned}
& L_{m}\left(\phi^{\prime}, S^{\prime}\right) \leq c^{2} \cdot L_{m}(\phi, S), \\
& L_{m}(\phi, S)=\max \left\{L\left(\phi^{m}\left(\gamma_{i}\right), S\right)\right\} \leq L_{m}\left(\phi^{\prime}, S^{\prime}\right) .
\end{aligned}
$$

This implies that $\operatorname{GR}\left(\phi^{\prime}\right)=\operatorname{GR}(\phi)$.

Suppose on the contrary, for any $m>0$, there is some $j$ such that $\phi^{m}\left(\delta_{j}\right) \neq 1$. Then $\max \left\{L\left(\phi^{m}\left(\delta_{j}\right), S\right)\right\}=1$. Hence

$$
\begin{aligned}
L_{m}(\phi, S) & =\max \left\{L\left(\phi^{m}\left(\gamma_{i}\right), S\right), L\left(\phi^{m}\left(\delta_{j}\right), S\right)\right\} \\
& =\max \left\{L\left(\phi^{m}\left(\gamma_{i}\right), S\right), 1\right\} \leq \max \left\{L_{m}\left(\phi^{\prime}, S^{\prime}\right), 1\right\} .
\end{aligned}
$$


This implies that $\operatorname{GR}\left(\phi^{\prime}\right) \leq \operatorname{GR}(\phi) \leq \max \left\{\operatorname{GR}\left(\phi^{\prime}\right), 1\right\}$. Since $\phi^{\prime}$ is not eventually trivial, Lemma 2.3 implies that $\operatorname{GR}\left(\phi^{\prime}\right) \geq 1$, and hence $\operatorname{GR}(\phi)=\operatorname{GR}\left(\phi^{\prime}\right)$.

Next we assume that $p \geq 1$. For each $j=1, \ldots, u$, we write $\phi\left(\delta_{j}\right)=\delta_{j_{1}} w_{1}$ for some $j_{1}$ and $w_{1} \in \pi^{\prime}$. Then

$$
\phi^{m}\left(\delta_{j}\right)=\delta_{j_{m}} w_{m} \phi\left(w_{m-1}\right) \cdots \phi^{m-1}\left(w_{1}\right),
$$

and thus

$$
L\left(\phi^{m}\left(\delta_{j}\right), S\right) \leq 1+p+p L_{1}\left(\phi^{\prime}, S^{\prime}\right)+p L_{2}\left(\phi^{\prime}, S^{\prime}\right)+\cdots+p L_{m-1}\left(\phi^{\prime}, S^{\prime}\right) .
$$

Let $L=\mathrm{GR}\left(\phi^{\prime}\right)$. By the assumption of our proposition, $L \geq 1$. Let $\epsilon>0$ be given. Since

$$
\lim _{m \rightarrow \infty} L_{m}\left(\phi^{\prime}, S^{\prime}\right)^{1 / m}=L,
$$

there is some $N>0$ such that if $m>N$, then $L_{m}\left(\phi^{\prime}, S^{\prime}\right)<(L+\epsilon)^{m}$. Choose $q_{1}, \ldots, q_{N}>0$ such that $L_{i}\left(\phi^{\prime}, S^{\prime}\right)<q_{i}(L+\epsilon)^{i}$ for $i=1, \ldots, N$. Put

$$
q=\max \left\{q_{1}, \ldots, q_{N}, 1\right\} \geq 1 .
$$

Then $L_{m}\left(\phi^{\prime}, S^{\prime}\right)<q(L+\epsilon)^{m}$ for all $m \geq 1$. Hence we have

$$
\begin{aligned}
L\left(\phi^{m}\left(\delta_{j}\right), S\right) & \leq 1+p+p q(L+\epsilon)+p q(L+\epsilon)^{2}+\cdots+p q(L+\epsilon)^{m-1} \\
& \leq 1+p q \frac{(L+\epsilon)^{m}-1}{(L+\epsilon)-1} .
\end{aligned}
$$

Since $p q \neq 0$, this implies that

$$
\lim _{m \rightarrow \infty} \sqrt[m]{\max _{j}\left\{L\left(\phi^{m}\left(\delta_{j}\right), S\right)\right\}} \leq L+\epsilon .
$$

Since $\pi^{\prime}$ is undistorted in $\pi$, there exists some $c>0$ such that

$$
\begin{aligned}
L_{m}\left(\phi^{\prime}, S^{\prime}\right) & =\max _{i}\left\{L\left(\phi^{\prime m}\left(\gamma_{i}\right), S^{\prime}\right)\right\} \\
& \leq c^{2} \cdot \max _{i, j}\left\{L\left(\phi^{\prime m}\left(\gamma_{i}\right), S\right), L\left(\phi^{m}\left(\delta_{j}\right), S\right)\right\}=c^{2} L_{m}(\phi, S),
\end{aligned}
$$

and hence we obtain

$$
L=\mathrm{GR}\left(\phi^{\prime}\right) \leq \mathrm{GR}(\phi)=\lim _{m \rightarrow \infty} \sqrt[m]{L_{m}(\phi, S)} \leq L+\epsilon
$$

for all $\epsilon>0$. Consequently, $\operatorname{GR}(\phi)=\operatorname{GR}\left(\phi^{\prime}\right)$.

Suppose that $\phi^{\prime}$ is an eventually trivial endomorphism of $\pi^{\prime}$. Then it is clear that $\operatorname{GR}\left(\phi^{\prime}\right)=0$. Consider a set $S=\left\{\gamma_{1}, \ldots, \gamma_{t}, \delta_{1}, \ldots, \delta_{u}\right\}$ of generators for $\pi$ 
as above. For any $m>0$, we observe that $\phi^{m}\left(\gamma_{i}\right)=1$ and $\phi^{m}\left(\delta_{j}\right)=\delta_{j_{m}} w_{m}$ for some $j_{m} \in\{1, \ldots, u\}$ and $w_{m}$ in a finite subset of $\pi^{\prime}$. This implies that the sequence $\left\{L_{m}(\phi, S)\right\}$ is bounded. Because $L_{m}(\phi, S)=0$ or $\geq 1$, it follows that $\operatorname{GR}(\phi)=0$ or 1 respectively.

When $\operatorname{GR}(\phi)=0$, Lemma 2.3 says that $\phi$ is an eventually trivial endomorphism. Next we consider the case when $\operatorname{GR}(\phi)=1$. From the definition, we can choose $N>0$ so that, for $m \geq N$, we have $1 / 2^{m}<L_{m}(\phi, S)$, which implies that $L_{m}(\phi, S) \geq 1$ because $L_{m}(\phi, S)$ is an integer. Therefore, for each $m \geq N$, we can choose $\gamma \in S$ such that $\phi^{m}(\gamma) \neq 1$. This shows that $\phi$ is not eventually trivial even though $\phi^{\prime}$ is eventually trivial.

Before leaving this section, we observe the following elementary fact.

Proposition 2.9. Let $\phi$ be an endomorphism of $\pi$ with a finite set $S$ of generators. Let

$$
\operatorname{GR}_{i}(\phi)=\lim _{k \rightarrow \infty} L\left(\phi^{k}\left(s_{i}\right), S\right)^{1 / k}
$$

for each $s_{i} \in S$. Then $\operatorname{GR}(\phi)=\max \left\{\operatorname{GR}_{i}(\phi) \mid s_{i} \in S\right\}$.

Proof. Since $L\left(\phi^{k}\left(s_{i}\right), S\right) \leq L_{k}(\phi, S)$, it follows that $\mathrm{GR}_{i}(\phi) \leq \mathrm{GR}(\phi)$. Assume $\operatorname{GR}_{i}(\phi)<\operatorname{GR}(\phi)$ for all $s_{i} \in S$. Thus there exists $K>0$ such that if $k \geq K$ and $s_{i} \in S$, then $L\left(\phi^{k}\left(s_{i}\right), S\right)^{1 / k}<\operatorname{GR}(\phi)$. Because $S$ is finite, it follows that $L_{k}(\phi, S)^{1 / k}<\mathrm{GR}(\phi)$ for all $k \geq K$. However, since

$$
\lim _{k \rightarrow \infty} L_{k}(\phi, S)^{1 / k}=\lim _{k \geq K} L_{k}(\phi, S)^{1 / k}=\inf _{k \geq K} L_{k}(\phi, S)^{1 / k},
$$

we obtain a contradiction: $\operatorname{GR}(\phi)=\inf _{k \geq K} L_{k}(\phi, S)^{1 / k}<\mathrm{GR}(\phi)$.

\section{Finitely generated nilpotent groups}

Consider the lower central series of a finitely generated group $\pi$,

$$
\pi=\pi_{1} \supset \pi_{2} \supset \cdots,
$$

where $\pi_{j}=\left[\pi, \pi_{j-1}\right]$ is the $j$-fold commutator subgroup $\gamma_{j}(\pi)$ of $\pi$. The endomorphism $\phi: \pi \rightarrow \pi$ induces endomorphisms

$$
\phi_{j}: \pi_{j} \rightarrow \pi_{j}, \quad \hat{\phi}_{j}: \pi / \pi_{j} \rightarrow \pi / \pi_{j}, \quad \bar{\phi}_{j}: \pi_{j} / \pi_{j+1} \rightarrow \pi_{j} / \pi_{j+1} .
$$

Then it is known from [2] that $\operatorname{GR}(\phi) \geq \operatorname{GR}\left(\bar{\phi}_{j}\right)^{1 / j}$ for all $j \geq 1$. The group $\pi$ is called nilpotent if $\pi_{j}=1$ for some $j$. When $\pi_{c} \neq 1$ but $\pi_{c+1}=1$, we say that it is $c$-step. 
Lemma 3.1 ([2, Proposition 2]). If $\pi$ is c-step nilpotent, then

$$
\operatorname{GR}(\phi)=\max \left\{\operatorname{GR}\left(\hat{\phi}_{c}\right), \operatorname{GR}\left(\phi_{c}\right)^{1 / c}\right\} .
$$

If $\pi$ is nilpotent, then

$$
\mathrm{GR}(\phi)=\max _{j \geq 1}\left\{\mathrm{GR}\left(\bar{\phi}_{j}\right)^{1 / j}\right\} .
$$

Recall, for example from [10, Proposition 3.1], that a finitely generated nilpotent group $\pi$ is virtually torsion-free. Thus there exists a finite-index, torsionfree, normal subgroup $\Gamma$ of $\pi$. Following the proof of [11, Lemma 3.1], we can see that there exists a fully invariant subgroup $\Lambda \subset \Gamma$ of $\pi$ which is of finite index. Therefore, any endomorphism $\phi: \pi \rightarrow \pi$ restricts to an endomorphism $\phi^{\prime}: \Lambda \rightarrow \Lambda$. By Theorem 2.8, we may consider only the case when $\phi^{\prime}$ is not eventually trivial, and hence we may assume that $\operatorname{GR}(\phi)=\operatorname{GR}\left(\phi^{\prime}\right)$. Consequently, for the computation of $\operatorname{GR}(\phi)$, we may assume that $\pi$ is a finitely generated torsion-free nilpotent group.

Consider the lower central series of a finitely generated torsion-free $c$-step nilpotent group $\pi$,

$$
\pi=\pi_{1}, \quad \pi_{j+1}=\left[\pi, \pi_{j}\right], \quad \pi_{c} \neq 1 \text { and } \pi_{c+1}=1 .
$$

For each $j=1, \ldots, c$, we consider the isolator of $\pi_{j}$ in $\pi$,

$$
\sqrt{\pi_{j}}=\sqrt[\pi]{\pi_{j}}:=\left\{x \in \pi \mid x^{k} \in \pi_{j} \text { for some } k \geq 1\right\} .
$$

Then it is known that $\sqrt{\pi_{j}}$ is a characteristic subgroup of $\pi$ with $\left[\sqrt{\pi_{j}}: \pi_{j}\right]$ finite. Furthermore, $\sqrt{\pi_{j}} / \pi_{j}$ is precisely the set of all torsion elements in the nilpotent group $\pi / \pi_{j}$, and $\sqrt{\pi_{j}} / \sqrt{\pi_{j+1}} \cong \mathbb{Z}^{k_{j}}$ for some integer $k_{j}>0$. Hence we obtain the adapted central series $[4$, p. 3]

$$
\pi=\sqrt{\pi_{1}} \supset \sqrt{\pi_{2}} \supset \cdots \supset \sqrt{\pi_{c}} \supset \sqrt{\pi_{c+1}}=1 .
$$

The following lemma plays a crucial role in our study of growth rates for endomorphisms of finitely generated nilpotent groups.

Lemma 3.2 ([15, Lemma 3.7]). Let $\pi$ be a finitely generated c-step nilpotent group with lower central series $\pi=\pi_{1} \supset \pi_{2} \supset \cdots \supset \pi_{c} \supset \pi_{c+1}=1$. Then there are finite sets $T_{j}=\left\{\tau_{j 1}, \ldots, \tau_{j k_{j}}\right\} \subset \pi_{j}$ such that

(1) if $p_{j}: \pi_{j} \rightarrow \pi_{j} / \pi_{j+1}$ denotes the projection, then $p_{j}\left(T_{j}\right)$ is an independent set of generators for the finitely generated abelian group $\pi_{j} / \pi_{j+1}$,

(2) if $j>1$, then every $\tau_{j r}$ is of the form $\left[\tau_{1 i}, \tau_{j-1, \ell}\right]$,

(3) $T_{1}$ generates $\pi$. 
Let $G$ be the Malcev completion of a finitely generated torsion-free nilpotent group $\pi$, and let $\phi$ be an endomorphism of $\pi$. Then $\phi$ extends uniquely to a Lie group homomorphism $D$ of $G$, called the Malcev completion of $\phi$. We call its differential $D_{*}$ the linearization of $\phi$.

Theorem 3.3. Let $\phi: \pi \rightarrow \pi$ be an endomorphism on a finitely generated torsionfree nilpotent group $\pi$. Let $G$ be the Malcev completion of $\pi$. Then the linearization $D_{*}:$ (S $\rightarrow$ (S) of $\phi$ can be expressed as a lower triangular block matrix with diagonal blocks $\left\{D_{j}\right\}$ so that

$$
\operatorname{GR}(\phi)=\max _{j \geq 1}\left\{\operatorname{sp}\left(D_{j}\right)^{1 / j}\right\}
$$

In particular, $\mathrm{GR}(\phi)$ is an algebraic integer.

Proof. Let $\pi$ be a finitely generated torsion-free $c$-step nilpotent group with the adapted central series

$$
\pi=\sqrt{\pi_{1}} \supset \sqrt{\pi_{2}} \supset \cdots \supset \sqrt{\pi_{c}} \supset \sqrt{\pi_{c+1}}=1 .
$$

Let $q_{j}: \sqrt{\pi_{j}} \rightarrow \sqrt{\pi_{j}} / \sqrt{\pi_{j+1}}$ denote the projection. We choose $\left\{T_{1}, \ldots, T_{c}\right\}$ as in Lemma 3.2. Since $\pi_{2}$ is a fully invariant, finite-index subgroup of $\sqrt{\pi_{2}}$, it induces a short exact sequence

$$
1 \rightarrow \sqrt{\pi_{2}} / \pi_{2} \rightarrow \pi_{1} / \pi_{2} \rightarrow \pi_{1} / \sqrt{\pi_{2}}=\sqrt{\pi_{1}} / \sqrt{\pi_{2}} \rightarrow 1 .
$$

Since $\sqrt{\pi_{2}} / \pi_{2}$ is finite, it follows that $\sqrt{\pi_{1}} / \sqrt{\pi_{2}} \cong \mathbb{Z}^{k_{1}}$ can be regarded as the free part of the finitely generated abelian group $\pi_{1} / \pi_{2}$. Hence we can choose $S_{1} \subset T_{1}$ such that $p_{1}\left(S_{1}\right)$ is an independent set of free generators of $\sqrt{\pi_{1}} / \sqrt{\pi_{2}}$ and $p_{1}\left(T_{1}-S_{1}\right)$ is an independent set of torsion generators of $\pi_{1} / \pi_{2}$.

Next we consider the short exact sequence

$$
1 \rightarrow \sqrt{\pi_{3}} / \pi_{3} \rightarrow \sqrt{\pi_{2}} / \pi_{3} \rightarrow \sqrt{\pi_{2}} / \sqrt{\pi_{3}} \rightarrow 1 .
$$

Since $\pi_{2} / \pi_{3} \subset \sqrt{\pi_{2}} / \pi_{3}$, we obtain the following commutative diagram between exact sequences:

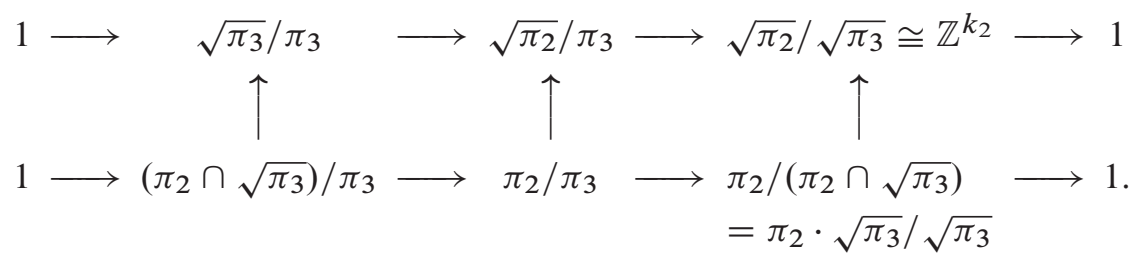


where all vertical maps are inclusions of finite index. So we can choose $S_{2} \subset T_{2}$ such that $p_{2}\left(S_{2}\right)$ is an independent set of free generators of the free abelian group $\left(\pi_{2} \cdot \sqrt{\pi_{3}}\right) / \sqrt{\pi_{3}}$ and $p_{2}\left(T_{2}-S_{2}\right)$ is an independent set of torsion generators of $\pi_{2} / \pi_{3}$. Note that $S_{2} \subset \pi_{2} \subset \sqrt{\pi_{2}}$. Because the right-most vertical inclusion is of finite index, we can choose $\mathbb{S}_{2} \subset \sqrt{\pi_{2}}$ such that $q_{2}\left(\mathbb{S}_{2}\right)$ is an independent set of free generators of $\sqrt{\pi_{2}} / \sqrt{\pi_{3}}$, and for each $\sigma_{2} \in \mathbb{S}_{2}$, there are unique $\ell_{2} \geq 1$ and unique $\tau_{2 *} \in S_{2}$ such that $\sigma_{2}{ }^{\ell_{2}}=\tau_{2 *}$ modulo $\sqrt{\pi_{3}}$. We remark also that $\# S_{2}=\# \mathbb{S}_{2}$.

Continuing in this way, we obtain $\left\{S_{1}, \ldots, S_{c}\right\} \subset\left\{T_{1}, \ldots, T_{c}\right\}$ such that

- $S_{j} \subset T_{j}, \# S_{j}=\# \mathbb{S}_{j}$,

- $p_{j}\left(S_{j}\right)$ is an independent set of free generators of $\pi_{j} / \pi_{j+1}$,

- $p_{j}\left(T_{j}-S_{j}\right)$ is an independent set of torsion generators of $\pi_{j} / \pi_{j+1}$,

- $q_{j}\left(\mathbb{S}_{j}\right)$ is an independent set of free generators of $\sqrt{\pi_{j}} / \sqrt{\pi_{j+1}}$,

- for each $\sigma_{j} \in \mathbb{S}_{j} \subset \sqrt{\pi_{j}}$, there exist unique $\ell_{j} \geq 1$ and $\tau_{j *} \in S_{j}$ such that

$$
\sigma_{j}^{\ell_{j}}=\tau_{j *} \bmod \sqrt{\pi_{j+1}} .
$$

The adapted central series of $\pi$ allows us to choose a preferred basis a of $\pi$; we can choose $\mathbf{a}$ to be $\left\{\mathbb{S}_{1}, \ldots, \mathbb{S}_{c}\right\}$ so that it generates $\pi$ and $\pi$ can be embedded as a lattice of a connected, simply connected nilpotent Lie group $G$, the Malcev completion of $\pi$. Its Lie algebra $\mathbb{S}$ has a linear basis $\log \mathbf{a}=\left\{\log \mathbb{S}_{1}, \ldots, \log \mathbb{S}_{c}\right\}$. From $\sigma_{j} \ell_{j}=\tau_{j *} \bmod \sqrt{\pi_{j+1}}$, we have

$$
\ell_{j} \log \left(\sigma_{j}\right)=\log \left(\sigma_{j}^{\ell_{j}}\right)=\log \left(\tau_{j *}\right) \bmod \gamma_{j+1}(\circlearrowleft) .
$$

This implies that $\left\{\log S_{1}, \ldots, \log S_{c}\right\}$ is also a linear basis of 5 .

Let $\phi: \pi \rightarrow \pi$ be an endomorphism. Then $\phi$ induces endomorphisms

$$
\phi_{j}: \pi_{j} \rightarrow \pi_{j}, \quad \hat{\phi}_{j}: \pi / \pi_{j} \rightarrow \pi / \pi_{j}, \quad \bar{\phi}_{j}: \pi_{j} / \pi_{j+1} \rightarrow \pi_{j} / \pi_{j+1}
$$

and

$$
\begin{gathered}
\varphi_{j}: \sqrt{\pi_{j}} \rightarrow \sqrt{\pi_{j}}, \quad \hat{\varphi}_{j}: \pi / \sqrt{\pi_{j}} \rightarrow \pi / \sqrt{\pi_{j}}, \\
\bar{\varphi}_{j}: \sqrt{\pi_{j}} / \sqrt{\pi_{j+1}} \rightarrow \sqrt{\pi_{j}} / \sqrt{\pi_{j+1}} .
\end{gathered}
$$

Moreover, any endomorphism $\phi$ on $\pi$ extends uniquely to a Lie group endomorphism $D$ on $G$, the Malcev completion of $\phi$. With respect to the preferred basis $\log \mathbf{a}$ of the Lie algebra $G$ of $G$, we can express the linearization $D_{*}$ of $\phi$ as a lower triangular block matrix; each diagonal block $D_{j}$ is an integer matrix representing the endomorphism $\bar{\varphi}_{j}: \sqrt{\pi_{j}} / \sqrt{\pi_{j+1}} \cong \mathbb{Z}^{k_{j}} \rightarrow \sqrt{\pi_{j}} / \sqrt{\pi_{j+1}} \cong \mathbb{Z}^{k_{j}}$. For 
details, we refer to [9] for example. When the new basis $\left\{\log S_{1}, \ldots, \log S_{c}\right\}$ is used instead of $\log \mathbf{a}$, the integer entries of block matrices $D_{j}$ will be changed to rational entries because of identities (B), but the eigenvalues of $D_{j}$ will be unchanged. This means that, whenever the eigenvalues of $D_{*}$ are concerned, we may assume that $\pi_{j} / \pi_{j+1}$ is torsion-free, or $\pi_{j}=\sqrt{\pi_{j}}$. Consequently, we may assume that $\operatorname{GR}\left(\bar{\phi}_{j}\right)=\operatorname{GR}\left(\bar{\varphi}_{j}\right)$. Since $\sqrt{\pi_{j}} / \sqrt{\pi_{j+1}} \cong \mathbb{Z}^{k_{j}}$, by taking the tensor product with $\mathbb{R}$, it is known that $\operatorname{GR}\left(\bar{\varphi}_{j}\right)=\operatorname{sp}\left(D_{j}\right)$. Thus $\operatorname{GR}\left(\bar{\phi}_{j}\right)=\operatorname{sp}\left(D_{j}\right)$. Now the theorem follows from Lemma 3.1.

Remark 3.4. From Theorem 3.3, it follows that the growth rate of any endomorphism on a finitely generated torsion-free nilpotent group is an algebraic integer. The question of determining groups for which the growth rate of a group endomorphism is an algebraic number was raised by R. Bowen in [2, p. 27].

Example 3.5. Let Nil be the 3-dimensional Heisenberg group. That is,

$$
\text { Nil }=\left\{\left[\begin{array}{ccc}
1 & x & z \\
0 & 1 & y \\
0 & 0 & 1
\end{array}\right] \mid x, y, z \in \mathbb{R}\right\} .
$$

Consider the subgroups $\Gamma_{k}, k \in \mathbb{N}$, of Nil,

$$
\Gamma_{k}=\left\{\left[\begin{array}{ccc}
1 & n & \frac{\ell}{k} \\
0 & 1 & m \\
0 & 0 & 1
\end{array}\right] \mid m, n, \ell \in \mathbb{Z}\right\} .
$$

These are lattices of Nil, and every lattice of Nil is isomorphic to some $\Gamma_{k}$. Let

$$
a_{1}=\left[\begin{array}{lll}
1 & 0 & 0 \\
0 & 1 & 1 \\
0 & 0 & 1
\end{array}\right], \quad a_{2}=\left[\begin{array}{lll}
1 & 1 & 0 \\
0 & 1 & 0 \\
0 & 0 & 1
\end{array}\right], \quad a_{3}=\left[\begin{array}{ccc}
1 & 0 & \frac{1}{k} \\
0 & 1 & 0 \\
0 & 0 & 1
\end{array}\right] .
$$

Then $S=\left\{a_{1}, a_{2}, a_{3}\right\}$ is a generating set of $\Gamma_{k}$ satisfying

$$
\left[a_{1}, a_{2}\right]=a_{3}^{-k},\left[a_{1}, a_{3}\right]=\left[a_{2}, a_{3}\right]=1,
$$

and in fact,

$$
\left[\begin{array}{ccc}
1 & n & \frac{\ell}{k} \\
0 & 1 & m \\
0 & 0 & 1
\end{array}\right]=a_{1}^{m} a_{2}^{n} a_{3}^{\ell}
$$


Let

$$
\pi=\Gamma_{k}=\left\langle a_{1}, a_{2}, a_{3} \mid\left[a_{1}, a_{2}\right]=a_{3}^{-k},\left[a_{1}, a_{3}\right]=\left[a_{2}, a_{3}\right]=1\right\rangle .
$$

Let $\pi^{\prime}=\left\langle a_{3}\right\rangle$ and $S^{\prime}=\left\{a_{3}\right\}$. Since $\left(a_{3}^{-k}\right)^{n^{2}}=\left[a_{1}^{n}, a_{2}^{n}\right]$, we have

$$
L\left(\left(a_{3}^{-k}\right)^{n^{2}}, S^{\prime}\right)=k n^{2} \quad \text { and } \quad L\left(\left(a_{3}^{-k}\right)^{n^{2}}, S\right)=4 n .
$$

Hence

$$
L\left(\left(a_{3}^{-k}\right)^{n^{2}}, S^{\prime}\right)>L\left(\left(a_{3}^{-k}\right)^{n^{2}}, S\right)
$$

for all $n$ with $n>4 / k$. It follows that $\pi^{\prime}$ is distorted.

Consider any endomorphism $\phi: \pi \rightarrow \pi$. Then $\phi$ must be of the form

$$
\phi\left(a_{1}\right)=a_{1}^{m_{11}} a_{2}^{m_{21}} a_{3}^{p}, \quad \phi\left(a_{2}\right)=a_{1}^{m_{12}} a_{2}^{m_{22}} a_{3}^{q}, \quad \phi\left(a_{3}\right)=a_{3}^{m_{11} m_{22}-m_{12} m_{21}} .
$$

We will compute $\operatorname{GR}(\phi)$. The lower central series of $\pi$ is $\pi=\pi_{1} \supset \pi_{2}=\left\langle a_{3}^{k}\right\rangle$, and its adapted central series is $\pi=\pi_{1} \supset \sqrt{\pi_{2}}=\left\langle a_{3}\right\rangle$. We observe that

$$
T_{1}=\left\{a_{1}, a_{2}, a_{3}\right\} \quad \text { and } \quad T_{2}=\left\{a_{3}^{k}\right\}
$$

are sets satisfying the conditions of Lemma 3.2. Then we can see that

$$
S_{1}=\left\{a_{1}, a_{2}\right\} \subset T_{1}, \quad S_{2}=\left\{a_{3}^{k}\right\} \subset T_{2} \quad \text { and } \quad \mathbb{S}_{1}=\left\{a_{1}, a_{2}\right\}, \quad \mathbb{S}_{2}=\left\{a_{3}\right\} .
$$

Furthermore, $\left\{\mathbb{S}_{1}, \mathbb{S}_{2}\right\}=\left\{a_{1}, a_{2}, a_{3}\right\}$ is a preferred basis for $\pi$. The linearization of $\phi$ with respect to this preferred basis has two integer blocks $D_{1}$ and $D_{2}$, where

$$
D_{1}=\left[\begin{array}{ll}
m_{11} & m_{12} \\
m_{21} & m_{22}
\end{array}\right], \quad D_{2}=\left[m_{11} m_{22}-m_{12} m_{21}\right]=\left[\operatorname{det}\left(D_{1}\right)\right]
$$

By Theorem 3.3, we have $\operatorname{GR}(\phi)=\max \left\{\operatorname{sp}\left(D_{1}\right), \operatorname{sp}\left(D_{2}\right)^{1 / 2}\right\}$. Let $\mu, v$ be the eigenvalues of $D_{1}$. Then

$$
\operatorname{GR}(\phi)=\max \{|\mu|,|\nu|, \sqrt{|\mu \nu|}\}=\max \{|\mu|,|\nu|\}=\operatorname{sp}\left(D_{1}\right) .
$$

In fact, we show in Theorem 3.7 that it is always the case that $\operatorname{GR}(\phi)=\operatorname{sp}\left(D_{1}\right)$.

We consider another example in which we obtain much information about linearizations of endomorphisms, and then we obtain an idea of proving the next result, Theorem 3.7 . 
Example 3.6. Consider a 2-step torsion-free nilpotent group $\pi$ generated by

$$
\tau_{1}, \tau_{2}, \tau_{3}, \sigma_{12}, \sigma_{13}
$$

satisfying the relations

$$
\begin{gathered}
{\left[\tau_{1}, \tau_{2}\right]=\sigma_{12}, \quad\left[\tau_{1}, \tau_{3}\right]=\sigma_{13}, \quad\left[\tau_{2}, \tau_{3}\right]=\sigma_{12}^{m} \sigma_{13}^{n},} \\
{\left[\tau_{i}, \sigma_{j k}\right]=\left[\sigma_{12}, \sigma_{13}\right]=1 .}
\end{gathered}
$$

Since $\pi_{2}=\left\langle\sigma_{12}, \sigma_{13}\right\rangle \cong \mathbb{Z}^{2}$ and $\pi / \pi_{2}=\left\langle\bar{\tau}_{1}, \bar{\tau}_{2}, \bar{\tau}_{3}\right\rangle \cong \mathbb{Z}^{3}$, it follows that the set $\left\{T_{1}, T_{2}\right\}=\left\{\tau_{1}, \tau_{2}, \tau_{3}, \sigma_{12}, \sigma_{13}\right\}$ satisfies the conditions of Lemma 3.2 and forms a preferred basis of our group $\pi$. Let $\phi$ be an endomorphism of $\pi$. A direct computation shows that if

$$
\phi\left(\tau_{i}\right)=\tau_{1}^{d_{1 i}} \tau_{2}^{d_{2 i}} \tau_{3}^{d_{3 i}} \bmod \pi_{2},
$$

i.e., if the first block of the linearization of $\phi$ is

$$
D_{1}=\left[\begin{array}{lll}
d_{11} & d_{12} & d_{13} \\
d_{21} & d_{22} & d_{23} \\
d_{31} & d_{32} & d_{33}
\end{array}\right]
$$

then, with $\sigma_{23}=\left[\tau_{2}, \tau_{3}\right]$, we have

$$
\begin{aligned}
& \phi\left(\sigma_{12}\right)=\sigma_{12}^{M_{33}} \sigma_{13}^{M_{23}} \sigma_{23}^{M_{13}}, \\
& \phi\left(\sigma_{13}\right)=\sigma_{12}^{M_{32}} \sigma_{13}^{M_{22}} \sigma_{23}^{M_{12}}, \\
& \phi\left(\sigma_{23}\right)=\sigma_{12}^{M_{31}} \sigma_{13}^{M_{21}} \sigma_{23}^{M_{11}},
\end{aligned}
$$

where $M_{i j}$ denote the $(i, j)$-minor of $D$. These yield a matrix

$$
K=\left[\begin{array}{lll}
M_{33} & M_{32} & M_{31} \\
M_{23} & M_{22} & M_{21} \\
M_{13} & M_{12} & M_{11}
\end{array}\right]=\bigwedge^{2}\left(D_{1}\right),
$$

the second exterior power of $D_{1}$. On the other hand, since $\sigma_{23}=\sigma_{12}^{m} \sigma_{13}^{n}$, we have

$$
\begin{aligned}
\phi\left(\sigma_{12}\right) & =\sigma_{12}^{M_{33}} \sigma_{13}^{M_{23}} \sigma_{23}^{M_{13}}=\sigma_{12}^{M_{33}+m M_{13}} \sigma_{13}^{M_{23}+n M_{13}}, \\
\phi\left(\sigma_{13}\right) & =\sigma_{12}^{M_{32}} \sigma_{13}^{M_{22}} \sigma_{23}^{M_{12}}=\sigma_{12}^{M_{32}+m M_{12}} \sigma_{13}^{M_{22}+n M_{12}}, \\
\phi\left(\sigma_{23}\right) & =\phi\left(\sigma_{12}\right)^{m} \phi\left(\sigma_{13}\right)^{n} \\
& =\sigma_{12}^{M_{31}} \sigma_{13}^{M_{21}} \sigma_{23}^{M_{11}}=\sigma_{12}^{M_{31}+m M_{11}} \sigma_{13}^{M_{21}+n M_{11}} .
\end{aligned}
$$


From (3.1) and (3.2), the second block of the linearization of $\phi$ is

$$
D_{2}=\left[\begin{array}{ll}
M_{33}+m M_{13} & M_{32}+m M_{12} \\
M_{23}+n M_{13} & M_{22}+n M_{12}
\end{array}\right] .
$$

Plugging (3.1) and (3.2) into (3.3), we have

$$
\left\{\begin{array}{l}
{\left[\begin{array}{l}
M_{31} \\
M_{21} \\
M_{11}
\end{array}\right]=m\left[\begin{array}{l}
M_{33} \\
M_{23} \\
M_{13}
\end{array}\right]+n\left[\begin{array}{l}
M_{32} \\
M_{22} \\
M_{12}
\end{array}\right] \quad \text { when }(m, n) \neq(0,0),} \\
{\left[\begin{array}{l}
M_{31} \\
M_{21}
\end{array}\right]=\left[\begin{array}{l}
0 \\
0
\end{array}\right]}
\end{array}\right.
$$

When $(m, n) \neq(0,0)$, because of (3.4), $K$ is column equivalent to the matrix $K^{\prime}$ with the zero third column, and then, by doing some row operations on $K^{\prime}$, we can see that $K^{\prime}$ is row equivalent to the matrix $K^{\prime \prime}$, where

$$
K \rightsquigarrow K^{\prime}=\left[\begin{array}{lll}
M_{33} & M_{32} & 0 \\
M_{23} & M_{22} & 0 \\
M_{13} & M_{12} & 0
\end{array}\right] \rightsquigarrow K^{\prime \prime}=\left[\begin{array}{cc:c}
M_{33}+m M_{13} & M_{32}+m M_{12} & 0 \\
M_{23}+n M_{13} & M_{22}+n M_{12} & 0 \\
\hdashline M_{13} & M_{12} & 0
\end{array}\right] .
$$

Thus the second block $D_{2}$ of the linearization $D_{*}$ is a block submatrix of $K^{\prime \prime}$. This is obtained by removing the row and column of $K^{\prime \prime}$ that are determined by (3.3) or by the relation $\left[\tau_{2}, \tau_{3}\right]=\sigma_{12}^{m} \sigma_{13}^{n}$. Note also that $K, K^{\prime}$ and $K^{\prime \prime}$ have the same eigenvalues which are 0 and the eigenvalues of $D_{2}$. When $(m, n)=(0,0)$, because of (3.4), we have

$$
K=\left[\begin{array}{cc:c}
M_{33} & M_{32} & 0 \\
M_{23} & M_{22} & 0 \\
\hdashline M_{13} & M_{12} & M_{11}
\end{array}\right]
$$

Thus $D_{2}$ of $D_{*}$ is a block submatrix of $K$, and $K$ has $M_{11}$ and the eigenvalues of $D_{2}$ as its eigenvalues.

On the other hand, if $\mu_{1}, \mu_{2}, \mu_{3}$ are the eigenvalues of $D_{1}$, as $K=\bigwedge^{2}\left(D_{1}\right)$, the eigenvalues of $K$ are $\mu_{i} \mu_{j}(i<j)$. Consequently, we have

$$
\operatorname{sp}\left(D_{1}\right)=\max _{i=1,2,3}\left\{\left|\mu_{i}\right|\right\} \geq \max _{i \neq j}\left\{\sqrt{\left|\mu_{i} \mu_{j}\right|}\right\}=\operatorname{sp}(K) \geq \operatorname{sp}\left(D_{2}\right)^{1 / 2} .
$$

This proves that $\operatorname{GR}(\phi)=\operatorname{sp}\left(D_{1}\right)$. 
The following result was proved in [10] when $\phi$ is an automorphism using the intrinsic polynomial structure of nilpotent groups. We will now improve [10, Theorem 1.2] from automorphisms to endomorphisms by using completely different arguments.

Theorem 3.7. Let $\phi: \pi \rightarrow \pi$ be an endomorphism on a finitely generated torsionfree nilpotent group $\pi$ with Malcev completion $D$. Then

$$
\operatorname{GR}(\phi)=\operatorname{GR}\left(\phi_{\mathrm{ab}}\right),
$$

where $\phi_{\mathrm{ab}}: \pi /[\pi, \pi] \rightarrow \pi /[\pi, \pi]$ be the endomorphism induced by $\phi$. Hence we have $\operatorname{GR}(\phi)=\operatorname{sp}\left(D_{1}\right) \leq \operatorname{sp}\left(D_{*}\right)$.

Proof. Let $\pi$ be $c$-step and choose a family of finite sets $\left\{T_{1}, \ldots, T_{c}\right\}$ satisfying the conditions of Lemma 3.2. As was observed in the proof of Theorem 3.3, we can choose $\left\{S_{1}, \ldots, S_{c}\right\}$ such that each $S_{j} \subset T_{j} \subset \pi_{j}$ projects onto free generators of $\pi_{j} / \pi_{j+1}$ and a preferred basis $\left\{\mathbb{S}_{1}, \ldots, \mathbb{S}_{c}\right\}$ of $\pi$ so that each block matrix $D_{j}$ of the linearization $D_{*}$ of $\phi$ which is determined by $\log \mathbb{S}_{j}$ may be assumed to be determined by $\log S_{j}$.

Indeed, for each $j$ with $1 \leq j \leq c$, we write $S_{j}=\left\{\tau_{j 1}, \ldots, \tau_{j k_{j}}\right\} \subset T_{j}$; then, if $j>1$, every $\tau_{j r}$ is of the form $\left[\tau_{1 i}, \tau_{j-1, \ell}\right]$. For $1 \leq j \leq c$, if

$$
\phi\left(\tau_{j \ell}\right)=\tau_{j 1}^{d_{1 \ell}^{j}} \cdots \tau_{j k_{j}}^{d_{k_{j} \ell}^{j}} \text { modulo } \pi_{j+1},
$$

then the $j$ th block of the linearization $D_{*}$ of $\phi$ is

$$
D_{j}=\left[\begin{array}{ccc}
d_{11}^{j} & \cdots & d_{1 k_{j}}^{j} \\
\vdots & & \vdots \\
d_{k_{1} 1}^{j} & \cdots & d_{k_{j} k_{j}}^{j}
\end{array}\right] .
$$

In order to compare first the eigenvalues of $D_{1}$ with those of $D_{2}$, we use the following new notation: $D_{1}=\left[d_{i j}^{1}\right]=\left[d_{i j}\right], \sigma_{i j}=\left[\tau_{1 i}, \tau_{1 j}\right]$ for all $1 \leq i<j \leq k_{1}$. Then $\sigma_{i j}=\tau_{2, \ell}^{ \pm 1} \in S_{2}$ for some $\ell$, or $\sigma_{i j}$ is a word of elements in $S_{2}^{ \pm 1}$ modulo $\pi_{3}$ (see the presentation of $\pi$ in Example 3.6). Let $S=\left\{\sigma_{i j} \mid 1 \leq i<j \leq k_{1}\right\}$; then we may assume that $S_{2} \subset S$. Further, $S_{2}$ differs from $S$ except possibly by $\sigma_{i j}$ 's, words of elements in $S_{2}^{ \pm 1}$ modulo $\pi_{3}$ (note in Example 3.6 that $S_{2}=\left\{\sigma_{12}, \sigma_{13}\right\}$ and $\left.S=\left\{\sigma_{12}, \sigma_{13}, \sigma_{12}^{m} \sigma_{13}^{n}\right\}\right)$.

Now we can express $\phi\left(\sigma_{i j}\right)$ as follows:

$$
\phi\left(\sigma_{i j}\right)=\sigma_{12}^{\boldsymbol{M}_{1,2}^{i, j}} \sigma_{13}^{\boldsymbol{M}_{1,3}^{i, j}} \cdots \sigma_{1 k_{1}}^{\boldsymbol{M}_{1, k_{1}}^{i, j}} \cdots \sigma_{k_{1}-1, k_{1}}^{\boldsymbol{M}_{k_{1}-1, k_{1}}^{i, j}} \text { modulo } \pi_{3}
$$


for some integers $M_{p, q}^{i, j}$. We denote by $K$ the $\left(\begin{array}{c}k_{1} \\ 2\end{array}\right) \times\left(\begin{array}{c}k_{1} \\ 2\end{array}\right)$ matrix $\left[M_{p, q}^{i, j}\right.$,

$$
K=\left[\begin{array}{cccc}
M_{1,2}^{1,2} & M_{1,2}^{1,3} & \cdots & M_{1,2}^{k_{1}-1, k_{1}} \\
M_{1,3}^{1,2} & M_{1,3}^{1,3} & \cdots & M_{1,3}^{k_{1}-1, k_{1}} \\
\vdots & \vdots & & \vdots \\
M_{k_{1}-1, k_{1}}^{1,2} & M_{k_{1}-1, k_{1}}^{1,3} & \cdots & M_{k_{1}-1, k_{1}}^{k_{1}-1, k_{1}}
\end{array}\right] .
$$

We will refer to the column vector $\left(M_{1,2}^{i, j}, M_{1,3}^{i, j}, \ldots, M_{1, k_{1}}^{i, j}, \ldots, M_{k_{1}-1, k_{1}}^{i, j}\right)^{t}$ of $K$ as the $(i, j)$-column of $K$. Note the following.

(i) For any $\sigma_{i j} \in S, M_{p, q}^{i, j}$ is unique for which $\sigma_{p q} \in S_{2}$.

(ii) If $\sigma_{i j} \in S-S_{2}$, then $\sigma_{i j}$ is a word $w$ of elements in $S_{2}^{ \pm 1}$ modulo $\pi_{3}$. If $w \neq 1$ modulo $\pi_{3}$, the $(i, j)$-column of $K$ is an integer combination of $(p, q)$-columns of $K$ corresponding to the elements $\sigma_{p q}$ appearing in the word $w$. If $w \equiv 1$, then $M_{p, q}^{i, j}=0$ for which $\sigma_{p q} \in S_{2}$.

(iii) The right-hand side of the expression $(\mathrm{P})$ can be rewritten in terms of only the elements of $S_{2}$ using the words $\sigma_{i j} \equiv w\left(\sigma_{p q}\right)$. This yields the second block $D_{2}$.

Since $\sigma_{i j}=\left[\tau_{1 i}, \tau_{1 j}\right]$, taking $\phi$ on both sides, we have (see [5, Lemma 4.1] or $[14$, p. 93, Lemma 4.1])

$$
\begin{aligned}
\sigma_{12}^{M_{1,2}^{i, j}} & \sigma_{13}^{M_{1,3}^{i, j}} \cdots \sigma_{1 k_{1}}^{M_{1, k_{1}}^{i, j}} \cdots \sigma_{k_{1}-1, k_{1}}^{M_{k_{1}-1, k_{1}}^{i, j}} \\
& =\left[\tau_{11}^{d_{1, i}} \cdots \tau_{1 k_{1}, i}^{d_{k_{1}, i}}, \tau_{11}^{d_{1, j}} \cdots \tau_{1 k_{1}, j}^{d_{k_{1}, j}}\right] \\
& =\prod_{p} \prod_{q}\left[\tau_{1 p}^{d_{p, i}}, \tau_{1 q}^{d_{q, j}}\right]=\prod_{p} \prod_{q}\left[\tau_{1 p}, \tau_{1 q}\right]^{d_{p, i} d_{q, j}} \\
& =\prod_{1 \leq p<q \leq k_{1}} \sigma_{p q}^{d_{p, i} d_{q, j}-d_{p, j} d_{q, i}} \operatorname{modulo} \pi_{3} .
\end{aligned}
$$

This shows that $K$ is the second exterior power of $D_{1}$, i.e., $K=\bigwedge^{2}\left(D_{1}\right)$. Hence, if $\mu_{i}\left(1 \leq i \leq k_{1}\right)$ are the eigenvalues of the matrix $D_{1}$, then $\mu_{i} \mu_{j}(i<j)$ are the eigenvalues of $K$.

From part (ii) of the above remarks, we see that $K$ is column equivalent to the matrix $K^{\prime}$ with zero $(i, j)$-column for which $\sigma_{i j}=w\left(\sigma_{p q}\right) \neq 1$ modulo $\pi_{3}$. We rearrange the elements of $S$ so that $S=S_{2} \cup\left(S-S_{2}\right)=S_{2} \cup S_{2}^{1} \cup S_{2}^{2}$, where $S_{2}^{1}=\left\{\sigma_{i j} \in S-S_{2} \mid \sigma_{i j}=1\right\}$ and $S_{2}^{2}=\left\{\sigma_{i j} \in S-S_{2} \mid \sigma_{i j} \neq 1\right\}$. Rearranging $S$ to $S_{2} \cup\left(S-S_{2}\right)$, we have

$$
K \sim_{C} K^{\prime}=\left[\begin{array}{c:c}
K^{\prime} & 0 \\
\hdashline * & \cdots \\
* & *
\end{array}\right] .
$$


The effect of part (iii) on $K$ and hence on $K^{\prime}$ is doing some row operations using the $(i, j)$-rows in the last block of $K^{\prime}$ for which $\sigma_{i j}=w\left(\sigma_{p q}\right) \neq 1$ modulo $\pi_{3}$. By rearranging $S$ further to $S_{2} \cup S_{2}^{1} \cup S_{2}^{2}$, we have

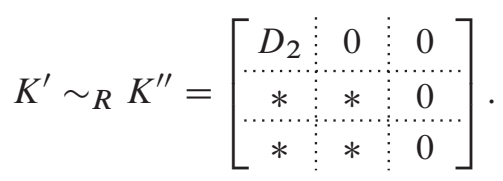

The middle block column is determined by the fact that if $\sigma_{i j} \equiv w \equiv 1$, then $M_{p, q}^{i, j}=0$ for which $\sigma_{p q} \in S_{2}$.

Consequently, the second block $D_{2}$ of $D_{*}$ is a block submatrix of $K^{\prime \prime}$ which is obtained by removing the rows and columns associated to $S-S_{2}$. Note also that $K, K^{\prime}$ and $K^{\prime \prime}$ have the same eigenvalues which contain the eigenvalues of $D_{2}$. This observation shows that

$$
\operatorname{sp}\left(D_{1}\right)=\max \left\{\left|\mu_{i}\right|\right\} \geq \max \left\{\sqrt{\mu_{i} \mu_{j}}\right\}=\operatorname{sp}(K)^{1 / 2} \geq \operatorname{sp}\left(D_{2}\right)^{1 / 2} .
$$

For the next inductive step, we recall that every element of $S_{3}\left(\subset T_{3}\right)$ is of the form $\left[\tau_{1 \ell}, \sigma_{i j}\right]$, where $i<j$. Taking $\phi$, we have

$$
\begin{aligned}
\phi\left(\left[\tau_{1 \ell}, \sigma_{i j}\right]\right) & =\left[\prod_{r} \tau_{1 r}^{d_{r, \ell}}, \prod_{1 \leq p<q \leq k_{1}} \sigma_{p q}^{d_{p, i} d_{q, j}-d_{p, j} d_{q, i}}\right] \\
& =\prod_{r} \prod_{1 \leq p<q \leq k_{1}}\left[\tau_{1 r}, \sigma_{p q}\right]^{d_{r, \ell}\left(d_{p, i} d_{q, j}-d_{p, j} d_{q, i}\right)} \text { modulo } \pi_{4} .
\end{aligned}
$$

This expression is unique except possibly for the exponents of the elements

$$
\left[\tau_{1 r}, \sigma_{p q}\right]=1 \text { modulo } \pi_{4} \text {. }
$$

This produces the matrix $K=D_{1} \otimes \bigwedge^{2} D_{1}$. First if $\left[\tau_{1 r}, \sigma_{p q}\right]=w\left(S_{3}\right) \neq 1$ modulo $\pi_{4}$, by doing some column operations and then by doing some row operations, we obtain a matrix $K^{\prime \prime}$, which can be regarded as a lower triangular block matrix. Finally, we remove the columns and rows from $K^{\prime \prime}$ which are associated with the elements $\left[\tau_{1 r}, \sigma_{p q}\right]=w\left(S_{3}\right)$ modulo $\pi_{4}$. This gives rise to the third block $D_{3}$ of $D_{*}$. Hence $\operatorname{sp}\left(D_{3}\right) \leq \operatorname{sp}\left(D_{1}\right)^{3}$. Continuing in this way, we may assume that the $j$ th block $D_{j}$ of $D_{*}$ is obtained from $\left(\otimes_{j-2} D_{1}\right) \otimes \bigwedge^{2} D_{1}$ so that

$$
\operatorname{sp}\left(D_{1}\right) \geq \operatorname{sp}\left(D_{j}\right)^{1 / j} .
$$

Consequently, $\operatorname{GR}(\phi)=\max \left\{\operatorname{sp}\left(D_{j}\right)^{1 / j}\right\}=\operatorname{sp}\left(D_{1}\right)=\operatorname{GR}\left(\phi_{\mathrm{ab}}\right) \leq \operatorname{sp}\left(D_{*}\right)$. 
Acknowledgments. The first-named author is indebted to the Max Planck Institute for Mathematics (Bonn) and Sogang University (Seoul) for the support and hospitality and the possibility of the present research during his visits there. The authors would like to thank the referee for their thorough reading and valuable comments on the original version of the article.

\section{Bibliography}

[1] M. Bestvina, M. Feighn and M. Handel, The Tits alternative for $\operatorname{Out}\left(F_{n}\right)$. I. Dynamics of exponentially-growing automorphisms, Ann. of Math. (2) 151 (2000), no. 2, 517-623.

[2] R. Bowen, Entropy and the fundamental group, in: The Structure of Attractors in Dynamical Systems (Fargo 1977), Lecture Notes in Math. 668, Springer, Berlin (1978), 21-29.

[3] T. C. Davis, Distortion in free nilpotent groups, Internat. J. Algebra Comput. 20 (2010), no. 5, 661-669.

[4] K. Dekimpe, Almost-Bieberbach Groups: Affine and Polynomial Structures, Lecture Notes in Math. 1639, Springer, Berlin, 1996.

[5] K. J. Falconer, B. Fine and D. Kahrobaei, Growth rate of an endomorphism of a group, Groups Complex. Cryptol. 3 (2011), no. 2, 285-300.

[6] M. Gromov, Asymptotic invariants of infinite groups, in: Geometric Group Theory. Vol. 2 (Sussex 1991), London Math. Soc. Lecture Note Ser. 182, Cambridge University, Cambridge (1993), 1-295.

[7] A. Katok and B. Hasselblatt, Introduction to the Modern Theory of Dynamical Systems, Encyclopedia Math. Appl. 54, Cambridge University, Cambridge, 1995.

[8] A. B. Katok, The entropy conjecture, in: Smooth Dynamical Systems (in Russian), Izdat. "Mir", (Moscow) (1977), 181-203.

[9] S. W. Kim and J. B. Lee, Anosov theorem for coincidences on nilmanifolds, Fund. Math. 185 (2005), no. 3, 247-259.

[10] T. Koberda, Entropy of automorphisms, homology and the intrinsic polynomial structure of nilpotent groups, in: In the Tradition of Ahlfors-Bers. VI, Contemp. Math. 590, American Mathematical Society, Providence (2013), 87-99.

[11] J. B. Lee and K. B. Lee, Lefschetz numbers for continuous maps, and periods for expanding maps on infra-nilmanifolds, J. Geom. Phys. 56 (2006), no. 10, 20112023.

[12] G. Levitt, Counting growth types of automorphisms of free groups, Geom. Funct. Anal. 19 (2009), no. 4, 1119-1146.

[13] G. Levitt and M. Lustig, Periodic ends, growth rates, Hölder dynamics for automorphisms of free groups, Comment. Math. Helv. 75 (2000), no. 3, 415-429. 
[14] D. S. Passman, The Algebraic Structure of Group Rings, Pure Appl. Math., WileyInterscience, New Yorky, 1977.

[15] J. A. Wolf, Growth of finitely generated solvable groups and curvature of Riemannian manifolds, J. Differential Geom. 2 (1968), 421-446.

Received June 16, 2020; revised July 12, 2020.

\section{Author information}

Alexander Fel'shtyn, Instytut Matematyki, Uniwersytet Szczecinski, ul. Wielkopolska 15, 70-451 Szczecin, Poland.

E-mail: fels@wmf .univ.szczecin.pl

Jang Hyun Jo, Department of Mathematics, Sogang University, Seoul 04107, Korea.

E-mail: jhjo@sogang.ac.kr

Corresponding author:

Jong Bum Lee, Department of Mathematics, Sogang University, Seoul 04107, Korea.

E-mail: jlee@sogang.ac.kr 